\title{
BROAD IRON LINES IN NEUTRONS STARS: DYNAMICAL BROADENING OR WIND SCATTERING?
}

\author{
Edward M. CaCKetT ${ }^{1}$ AND Jon M. Miller ${ }^{2}$ \\ ${ }^{1}$ Department of Physics and Astronomy, Wayne State University, 666 W. Hancock Street, Detroit, MI 48201, USA; ecackett@wayne.edu \\ ${ }^{2}$ Department of Astronomy, University of Michigan, 500 Church Street, Ann Arbor, MI 48105, USA \\ Received 2013 February 28; accepted 2013 August 19; published 2013 October 15
}

\begin{abstract}
Broad iron emission lines are observed in many accreting systems from black holes in active galactic nuclei and $\mathrm{X}$-ray binaries to neutron star low-mass X-ray binaries. The origin of the line broadening is often interpreted as due to dynamical broadening and relativistic effects. However, alternative interpretations have been proposed, included broadening due to Compton scattering in a wind or accretion disk atmosphere. Here we explore the observational signatures expected from broadening in a wind, in particular that the iron line width should increase with an increase in the column density of the absorber (due to an increase in the number of scatterings). We study the data from three neutron star low-mass X-ray binaries where both a broad iron emission line and absorption lines are seen simultaneously, and show that there is no significant correlation between line width and column density. This favors an inner disk origin for the line broadening rather than scattering in a wind.
\end{abstract}

Key words: accretion, accretion disks - stars: neutron - X-rays: binaries

Online-only material: color figures

\section{INTRODUCTION}

$\mathrm{X}$-ray emission from compact objects can often be characterized as a mixture of two direct emission components: thermal emission from an accretion disk (and stellar surface, in the case of neutron stars), and non-thermal emission that often takes a power-law form. The non-thermal or "hard" emission is attributed to a "corona." A short and incomplete list of viable models for the corona includes: thermal Comptonization in the innermost accretion flow (e.g., Sunyaev \& Titarchuk 1980), magnetic flares above the inner disk (e.g., Beloborodov 1999), and a mixture of processes in the base of a relativistic jet (e.g., Markoff et al. 2001).

Regardless of the specific form of the corona, however, hard $\mathrm{X}$-ray irradiation of the inner accretion disk is inevitable, and the reaction or "reflection" spectrum has been calculated in detail (e.g., George \& Fabian 1991). The most prominent feature of reflection is a broad iron emission line. Theoretical treatments of disk reflection have advanced continually, largely driven by observational results. Spectroscopic features consistent with the $\mathrm{X}$-ray irradiation of the inner accretion disk have now been observed in stellar-mass black holes, massive black holes in active galactic nuclei, and X-ray binaries harboring neutron stars (for reviews and summary papers, see, e.g., Miller 2007; Nandra et al. 2007; Cackett et al. 2010).

Disk reflection spectra are calculated in the fluid frame of the disk, and must be convolved with a relativistic blurring function to fit the spectra that are observed (for recent examples, see Brenneman \& Reynolds 2006; Dauser et al. 2010). The full power of disk reflection is then harnessed: the extent to which the spectra are blurred by Doppler shifts in the inner disk, and gravitational redshifts near to the compact object, can be used to measure the inner radius of the disk and black hole spin (e.g., Miller et al. 2009a; Brenneman \& Reynolds 2006; Walton et al. 2013, and references therein). In neutron star X-ray binaries, the inner extent of the disk can be used to set a limit on the stellar radius (e.g., Cackett et al. 2008, 2010), and magnetic field (Cackett et al. 2009; Papitto et al. 2009; Miller et al. 2011).

The basic framework of these measurements and models, and related assumptions about the geometry of the inner accretion flow, have been subjected to rigorous tests and potential alternative interpretations (see, e.g., Misra \& Sutaria 1999; Reynolds \& Wilms 2000; Miller et al. 2009b; Reynolds et al. 2009). The recent detection of reverberation lags in time-resolved spectra of Seyferts is a strong confirmation of disk reflection spectroscopy in massive black holes (e.g., Fabian et al. 2009; Emmanoulopoulos et al. 2011; Zoghbi et al. 2012, 2013; De Marco et al. 2013; Cackett et al. 2013; Kara et al. 2013a, 2013b). Reverberation lags have also been seen in a stellar-mass black hole (Uttley et al. 2011) and there are lags in neutron star lowmass X-ray binaries even with hints of reverberation (Barret 2013).

The fact that reflection is seen in the hard X-ray states of stellar-mass black holes, in which winds are absent or unimportant (e.g., Miller et al. 2008, 2012; Ponti et al. 2012), again signals that the spectra originate through reflection, not through an alternative process, such as scattering in a wind (e.g., Laurent \& Titarchuk 2007; Titarchuk et al. 2009; Sim et al. 2008, 2010a, 2010b).

The case of neutron stars may be more complicated. Whereas the absence of a strong corona in black holes prevents strong disk reflection, hot blackbody emission from the stellar surface or boundary layer can drive disk reflection in neutron star lowmass X-ray binaries (Cackett et al. 2010; D’Aì et al. 2010). Thus, reflection can occur in much softer states in neutron stars, and potentially when winds are important. The question arises, then, if scattering in a wind or disk atmosphere might shape broad lines in neutron stars, rather than dynamics in the inner accretion disk. Based on recent observations of GX 13+1, for instance, Díaz Trigo et al. (2012) suggest a wind origin for the breadth of iron emission lines in neutron stars as a class. In this work, we critically examine the properties of disk winds and atmospheres, and broad iron emission lines, in three neutron star low-mass X-ray binaries.

\subsection{Observational Signatures of Broadening of Lines through Scattering in a Wind}

To assess whether disk winds are the source of line broadening in neutron star low-mass X-ray binaries we need to consider 
Table 1

Fe Emission Line Width and Equivalent Hydrogen Column Density

\begin{tabular}{lccc}
\hline \hline Object & $\begin{array}{c}\text { Emission Line } \\
\text { Width, } \sigma(\mathrm{keV})\end{array}$ & $\begin{array}{c}N_{\mathrm{H}} \\
\left(10^{22} \mathrm{~cm}^{-2}\right)\end{array}$ & Reference \\
\hline GX 13+1 & $0.88 \pm 0.10$ & $17.2 \pm 8.5$ & Díaz Trigo et al. (2012) \\
& $0.77 \pm 0.19$ & $6.6 \pm 3.0$ & \\
& $0.72 \pm 0.10$ & $6.6 \pm 1.5$ & \\
& $0.78 \pm 0.13$ & $6.0 \pm 4.3$ & \\
& $0.77 \pm 0.07$ & $7.1 \pm 1.4$ & \\
4U 1323-62 & $0.85 \pm 0.21$ & $3.6 \pm 1.0$ & Boirin et al. (2005) \\
& $0.55 \pm 0.32$ & $6.0 \pm 3.5$ & \\
4U 1624-490 & $0.47 \pm 0.32$ & $14.0 \pm 8.5$ & \\
& $0.50 \pm 0.23$ & $42.3 \pm 38.2$ & Xiang et al. (2009) \\
& $0.18 \pm 0.06$ & $26.7 \pm 15.7$ & \\
\hline
\end{tabular}

the observational signatures of such a scenario. As a photon passes through an accretion disk wind or corona, it will be Compton scattered, leading to a shift in energy of the photon, and hence a broadening of any emission line feature. The total number of scatterings determines the overall shift in energy for a given photon. Hence, an increase in the average number of scatterings per photon leads to an increase in the breadth of an emission line. The number of scatterings is set by both the electron temperature and the optical depth of the wind/corona. Therefore, for a given electron temperature, an increase in the optical depth will correspond to an increase in the width of an emission line. Observationally, then, we could expect to see an increase in the measured emission line width $(\sigma)$ with an increase in the measured warm absorption column density, when both emission and absorption lines are observed simultaneously. Such a correlation is claimed in GX $13+1$ by Díaz Trigo et al. (2012), and we study this further, below.

Díaz Trigo et al. (2012) also claim that an increase in the emission line equivalent width (EW) with a corresponding increase in the EW of the absorption line (or similarly the warm absorption column density) is a signature that the broad line width is determined by scattering. However, if the broad emission line flux is nearly constant and absorption increases, this reduces the continuum flux and hence leads to the EW of the emission and absorption lines correlating. Hence, such a correlation is not a clear observational signature of scattering. We discuss the strength of this effect in more detail below.

Here, we use values of the emission line width and absorption column density from the literature for three objects where both an $\mathrm{Fe} \mathrm{K}$ emission line and $\mathrm{Fe}$ absorption lines are observed simultaneously.

\section{COMPARISON OF LINE WIDTH AND ABSORPTION COLUMN}

We searched the literature for neutron star low-mass X-ray binaries that display both a broad iron emission line and iron absorption lines seen simultaneously. We also require multiple measurements of the line width and absorption column density in order to look for correlations between the two parameters. This resulted in data from three objects: GX 13+1, 4U 1323-62 and $4 \mathrm{U} 1624-490$. GX $13+1$ is one of only two neutron star low-mass X-ray binaries where significantly blue-shifted absorption lines have been detected (Ueda et al. 2004; Díaz Trigo et al. 2012), while 4U 1323-62 and 4U 1624-490 are both well-known dipping sources (e.g., Boirin et al. 2005; Xiang et al. 2009, and references therein) where their near edge-on inclination leads to strong dips in the lightcurve due to absorbing

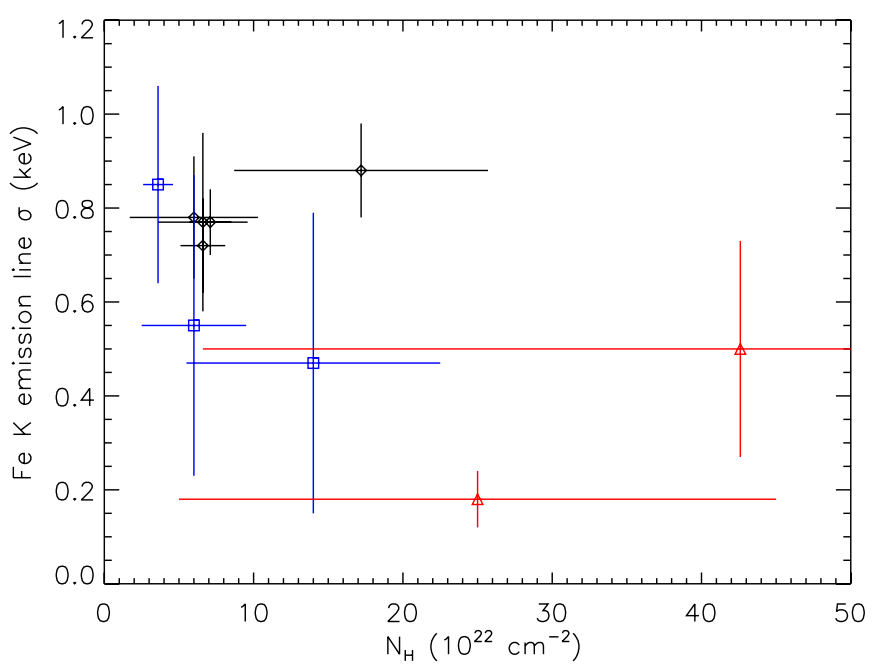

Figure 1. Equivalent hydrogen column density for the warm absorber $\left(N_{\mathrm{H}}\right)$ vs. iron emission line width $(\sigma)$ for GX 13+1 (black, diamonds), 4U 1323-62 (blue, squares) and 4U 1624-490 (red, triangles).

(A color version of this figure is available in the online journal.)

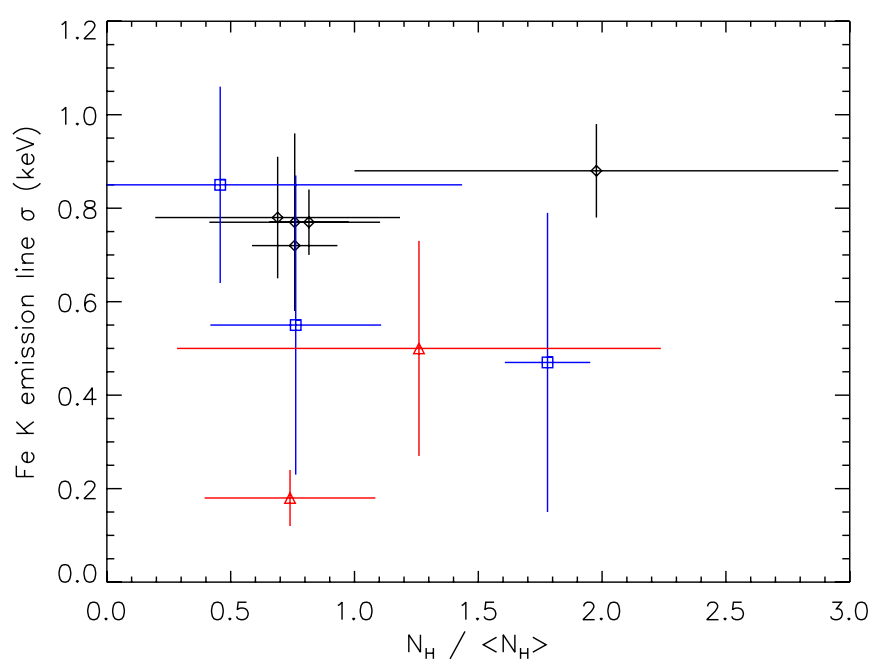

Figure 2. Normalized equivalent hydrogen column density for the warm absorber, $N_{\mathrm{H}} /\left\langle N_{\mathrm{H}}\right\rangle$, vs. iron emission line width $(\sigma)$ for GX $13+1$ (black, diamonds), 4U 1323-62 (blue, squares) and 4U 1624-490 (red, triangles).

(A color version of this figure is available in the online journal.)

material close to the accretion disk (e.g., Frank et al. 1987). Note that GX $13+1$ is also likely a dipping source (Diaz Trigo et al. 2010) and so these two sources are a good comparison. Sources need to be close to edge-on for both emission and absorption lines to be observed.

We summarize the data we use in Table 1, here we take the data directly from the papers, yet make the error bars symmetrical by taking the average (note that this does not affect the correlation tests which do not use the errors bars). For $4 \mathrm{U}$ 1624-490, Xiang et al. (2009) fit two absorbers, one warm and one hot. Here, we sum the two column densities to determine the total absorbing column, taking the mid-point of the uncertainty range. In Figure 1 we plot the absorbing column for the warm absorber versus the iron emission line width for these three sources. To be able to more directly compare the three objects, in Figure 2 we normalize the column densities by the mean column density for each object. As can be seen from these figures, there is no overall trend of increasing emission line width with increasing column density. We test this with a Spearman's Rank 


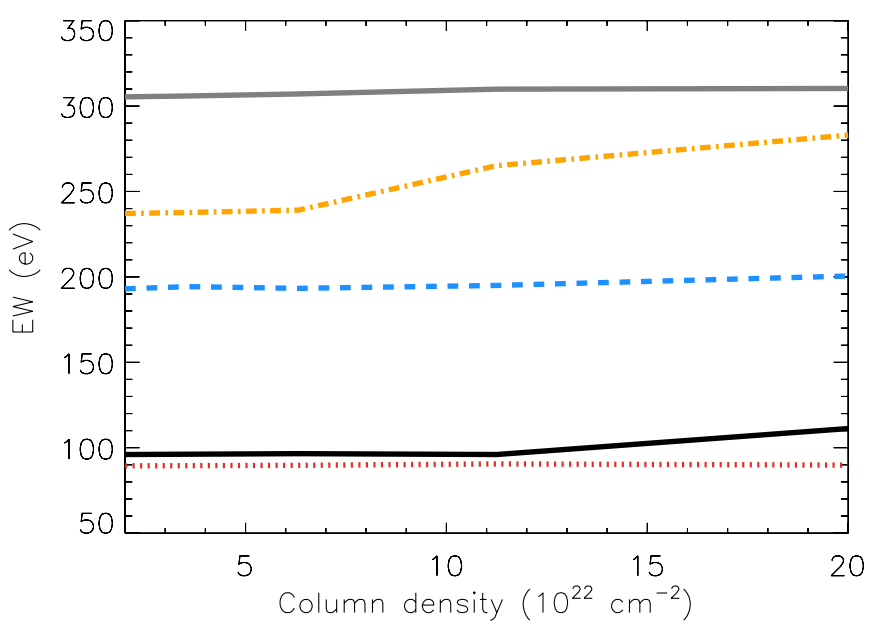

Figure 3. Emission line equivalent width vs. warm absorber column density for the continuum and line parameters found in GX 13+1 by Díaz Trigo et al. (2012). Each line represents the continuum/line parameters for different observations: observations 4 (gray, dash-triple dotted line), 6 (black, solid line), 7 (red, dotted), 8 (blue, dashed) and 9 (orange, dash-dotted). A clear increase in emission line EW with increasing column density is seen for two of the five continuum/line models.

(A color version of this figure is available in the online journal.)

correlation. Comparing all the column densities and line widths gives $r=-0.49$ (a weak anti-correlation), which corresponds to a $15 \%$ probability that the anti-correlation is not significant. If we instead compare the normalized column densities versus line width, we get $r=-0.14$, which corresponds to a $71 \%$ probability that the correlation is not significant. Hence, there is no significant correlation when comparing all three sources.

The only source where there are enough data points to test for a correlation individually is GX $13+1$. The Spearman's Rank correlation for GX $13+1$ is $r=0.29$ which corresponds to a $64 \%$ probability of no significant correlation. We further look to see if there is a significant correlation in GX $13+1$ by fitting a straight line to the data, using errors in both parameters (utilizing the fitexy routine in IDL). This leads to a best-fitting line with a slope of $0.012 \pm 0.016$, consistent with constant emission line width at the $1 \sigma$ level.

We therefore conclude that there is no significant correlation between emission line width and absorbing column density either globally for the three sources or for GX $13+1$ alone.

\section{ON CORRELATIONS BETWEEN EMISSION LINE EQUIVALENT WIDTH AND ABSORPTION COLUMN DENSITY}

Any increase in the absorption column density will lead to stronger absorption lines but also change the continuum flux. If the absorption is strong enough it will effect the continuum level through the Fe $\mathrm{K}$ band and hence change the measured $\mathrm{EW}$ of the $\mathrm{Fe} \mathrm{K}$ emission line even if the emission line flux remains constant. Thus, comparing absorption column density (or absorption line EW) with the emission line EW could lead to false correlations.

In order to quantify this effect, we use a model for warm absorption and change the column density to see how it affects the measured $\mathrm{EW}$ of an $\mathrm{Fe} \mathrm{K}$ emission line with constant line flux. We used an XSTAR grid of models calculated with $n=10^{13} \mathrm{~cm}^{-3}$ and a turbulent velocity of $300 \mathrm{~km} \mathrm{~s}^{-1}$. We also assumed the average luminosity for the source with an

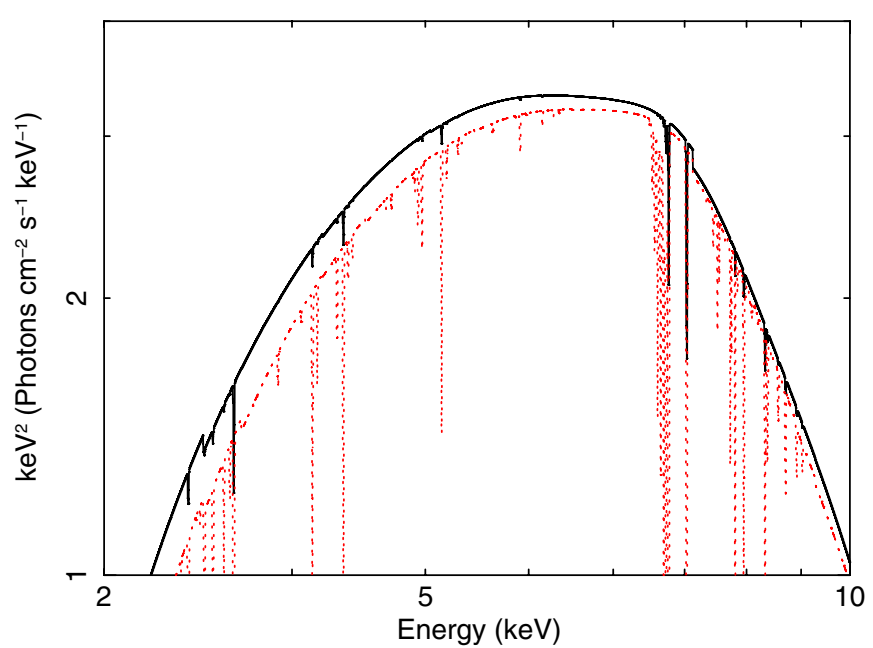

Figure 4. Model for observation 9 from Díaz Trigo et al. (2012) with the column density of the warm absorber set to $2 \times 10^{22} \mathrm{~cm}^{-2}$ (solid, black line) and $2 \times 10^{23} \mathrm{~cm}^{-2}$ (dotted, red line). A clear change in continuum strength through the Fe $\mathrm{K}$ band is seen.

(A color version of this figure is available in the online journal.)

irradiating spectrum comprised of a blackbody with $k T=2 \mathrm{keV}$. We use XSTAR directly rather than using the warmabs model within xspec so that we can calculate the re-emission spectrum also (see next section).

We adopt the continuum and emission line parameters found for GX 13+1 by Díaz Trigo et al. (2012, their Table 4) and use our XSTAR model for the warm absorber component. We change only the column density of the warm absorber and measure the EW of the emission line at each value of $N_{\mathrm{H}}$. In Figure 3 we show how the emission line EW changes with $N_{\mathrm{H}}$ for the continuum and emission line parameters used in each of the five observations of GX 13+1 considered (Díaz Trigo et al. 2012).

The results show that a change in the measured emission line EW with increasing $N_{\mathrm{H}}$ depends on the continuum parameters. However, two of the five models show a clear overall increase in EW when $N_{\mathrm{H}}$ increases from $2 \times 10^{22} \mathrm{~cm}^{-2}$ to $2 \times 10^{23} \mathrm{~cm}^{-2}$. While several change by just a few $\mathrm{eV}$, the size of the increase in the two clearest is $46 \mathrm{eV}$ and $15 \mathrm{eV}$. This can explain some but not all of the change in EW observed in GX $13+1$ and intrinsic changes must also be involved. However, it highlights that a correlation between absorption column density or absorption line EW and emission line EW does not imply that the line is broadened by scattering and can arise simply due to the change in continuum caused by a change in absorption. We show the change in the model for observation 9 of Díaz Trigo et al. (2012) if we assume a warm absorber with $N_{\mathrm{H}}=2 \times 10^{22} \mathrm{~cm}^{-2}$ compared to $N_{\mathrm{H}}=2 \times 10^{23} \mathrm{~cm}^{-2}$ in Figure 4 where a clear change in the continuum strength can be seen.

Furthermore, we can test the statistical significance of the correlation between absorption line EW and emission line EW or between column density and emission line EW in GX 13+1. The strongest absorption line, and the only absorption line to be detected in all five observations considered in Díaz Trigo et al. (2012) is Fe XXVI K $\alpha$. Comparing the Fe XXVI K $\alpha$ absorption line EW with the Fe emission line EW (their Table 3) we get $r=0.60$ and a $28 \%$ probability that the correlation is false. If we instead compare the warm absorber column density and Fe emission line EW (their Table 4) we get $r=0.66(22 \%$ probability). In other words, there is no statistically significant correlation. 


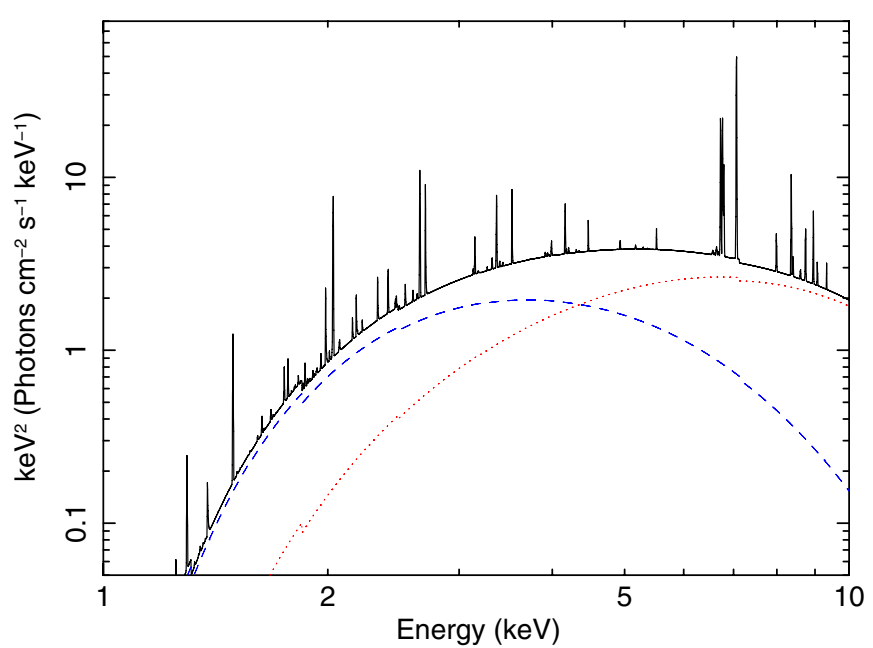

Figure 5. Spectrum re-emitted by an accretion disk wind/atmosphere. This is based on assuming $n=10^{13} \mathrm{~cm}^{-3}$ and using the warm absorber parameters for observation 6 in Díaz Trigo et al. (2012). We also assume the continuum model for observation 6 (disk blackbody is shown by a blue dashed line while the blackbody is shown by a red dotted line). The relative normalization of the continuum and emission line spectrum depends on the specific geometry. Here we have set it so that the $6.7 \mathrm{keV} \mathrm{Fe}$ line has the same line flux as quoted in Table 4 of Díaz Trigo et al. (2012).

(A color version of this figure is available in the online journal.)

\section{ON THE EMISSION SPECTRUM FROM A WIND}

X-ray flux absorbed in the accretion disk wind/corona will be re-emitted, leading the emission lines. We show an example of such an emission spectrum in Figure 5, where we use the same XSTAR models calculated above to determine the emission spectrum. We used the warm absorber parameters for observation 6 in Díaz Trigo et al. (2012). The relative normalization of the continuum and emission line spectrum depends on the specific geometry. Here we have set it so that the $6.7 \mathrm{keV}$ Fe line has the same line flux as quoted in Table 4 of Díaz Trigo et al. (2012). Intrinsically narrow emission lines are produced across the spectrum.

Although the emission lines are intrinsically narrow, they will be broadened by Comptonization as well as due to Doppler broadening at the velocities in the outer disk. Here, we consider whether Compton broadening in a disk atmosphere is sufficient to give the broad lines observed in GX $13+1$. We want to consider what are the realistic conditions in the disk atmosphere/corona and what broadening that will lead to. The change in energy per Compton scattering goes like $d E / E=k T_{e} / m_{e} c^{2}$, thus it is important to determine a realistic electron temperature. One of the most detailed studies of an extended accretion disk corona is that of Jimenez-Garate et al. (2005) who apply detailed photoionization models to a high quality Chandra HETGS spectrum of the neutron star low-mass X-ray binary (LMXB) Her X-1. They find a temperature of $k T=7 \mathrm{eV}\left(8 \times 10^{4} \mathrm{~K}\right)$ based on the Ne IX line and narrow lines $\Delta v \leqslant 260 \mathrm{~km} \mathrm{~s}^{-1}$. A much more extreme example is the black hole X-ray binary GRO J1655-40 which showed a powerful outflowing wind. The electron temperature there is determined to be $(0.2-1.0) \times 10^{6} \mathrm{~K}$ (Miller et al. 2006). If we conservatively assume that $k T$ is factor of 14 higher than Her X-1 and comparable to the extreme case of GRO J1655-40, say $0.1 \mathrm{keV}$, then $d E / E=2 \times 10^{-4}$, which for a $6.7 \mathrm{keV}$ emission line is $1.3 \times 10^{-3} \mathrm{keV}$ per scatter. We can calculate the optical depth, $\tau$, of the warm absorber from the column density via $\tau=N \sigma_{T}$, where $\sigma_{T}$ is the Thomson crosssection $=6.65 \times 10^{-25} \mathrm{~cm}^{2}$. The largest warm absorber column density for GX 13+1 measured in Díaz Trigo et al. (2012) is $1.7 \times 10^{23} \mathrm{~cm}^{-2}$, thus, we get $\tau=0.11$. The optical depth is therefore not high enough to give enough scatterings to lead to a line as broad as seen in GX $13+1$.

\section{DISCUSSION}

Recent observations of the neutron star LMXB GX 13+1 have been suggested as evidence that the origin of broad emission lines in neutron star low-mass X-ray binaries is due scattering in disk winds (Díaz Trigo et al. 2012). Here, we have looked at three neutron star low-mass X-ray binaries where both a broad iron emission line and absorption lines have been observed simultaneously. If the breadth of the iron emission line is set by scattering in the disk wind or corona, then one should expect a correlation between the width of the emission line and the absorbing column - a larger absorbing column leads to more scattering and hence a broader line. By comparing these two quantities in three neutron stars we find no statistically significant correlation either globally, or on an individual basis for GX $13+1$. We therefore conclude that the observational evidence does not indicate broadening due to scattering in a disk wind.

One complication that we are not able to test is that a change in electron temperature in the disk wind/corona will change the emission line width-the electron temperature sets the energy shift per scatter. While we cannot account for this in our analysis, we can point to the fact that none of the objects show a significant change in line width between different spectra.

Díaz Trigo et al. (2012) claim that in GX 13+1 an increase in emission line EW is correlated with the absorption line EW and that this implies the broad emission line is due to scattering. Firstly, there is no statistically significant correlation between the EWs, or the emission line EW and column density. Furthermore, a correlation between EWs does not necessarily imply line broadening as a change in continuum strength due to absorption can lead to a change in EW, though this depends on the continuum shape and warm absorber parameters.

Another insight into the origin of broad iron lines in neutron star low-mass X-ray binaries can be gleaned by considering magnetic field constraints that have recently been achieved in accreting X-ray pulsars. Broad, likely relativistic iron lines in SAX J1808.6-3658 (Cackett et al. 2009; Papitto et al. 2009) and IGR J17480-2446 (in Terzan 5; Miller et al. 2011) have placed limits on the inner radial extent of the accretion disk. In both cases, assuming that the disk is truncated by the stellar magnetic dipole gives a field strength equivalent to estimates based on $\mathrm{X}$-ray timing traces of the inner disk extent. These examples of correspondence clearly signal that the iron emission line width is driven by dynamics in the inner disk, not by scattering in a distant wind.

Further support of reflection in neutron star low-mass X-ray binaries is the correspondence between blackbody flux and Fe $\mathrm{K}$ emission line flux in 4U 1705-44 (Lin et al. 2010) during the soft state. This demonstrates that during soft states the blackbody component (likely associated with the neutron star boundary layer) can be irradiating the accretion disk leading to reflection. Softer states in neutron star low-mass X-ray binaries do not necessarily have weaker reflection than harder states, and the source of the X-ray irradiation in soft and hard states is likely different (Cackett et al. 2010; D’Aì et al. 2010). 
Another point of comparison is the so-called accretion disk corona sources, wherein the line of sight is thought to more persistently intercept the disk atmosphere. A good example may be 2S 0921-63 as observed with the Chandra/HETGS. The spectrum is extremely hard $(\Gamma \simeq 1.1)$, and thus likely the result of scattering rather than direct emission. A number of emission lines are observed, including $\mathrm{Fe} \mathrm{K}$, with a measured width of $\sigma \leqslant 0.005 \mathrm{keV}$ (Kallman et al. 2003). Of course, this line is far narrower than any that would be plausibly associated with the inner disk, but it is consistent with theoretical treatments of disk atmospheres (e.g., Jimenez-Garate et al. 2001; see below).

Her X-1 may be another relevant example to consider in this context. The disk corona in this system has been studied extensively using gratings X-ray spectroscopy, and detailed analysis has succeeded in even constraining the electron number density of the gas (Jimenez-Garate et al. 2005). In the "low" state observed using the Chandra/HETGS, for instance, neutral, Helike, and H-like lines are detected. Each line has a width less than $\sigma \leqslant 680 \mathrm{~km} \mathrm{~s}^{-1}$ (or $\sigma \leqslant 0.02 \mathrm{keV}$; Jimenez-Garate et al. 2005). As we show here, even an electron density of more than 10 times that seen in Her X-1 is not high enough to give sufficient Compton broadening to match the Fe $\mathrm{K}$ emission line width in GX 13+1 given the observed warm absorber column densities.

Last, if a dense wind is the fundamental requirement for the production of broad iron lines, then such lines should also be detected in the spectra of high-mass X-ray binaries. Fürst et al. (2011) present a detailed study of the neutron star HMXB GX 301-2. The absorber is found to approach $N_{\mathrm{H}} \simeq 10^{24} \mathrm{~cm}^{-2}$, but the width of the iron emission line is just $\sigma=0.03-0.04 \mathrm{keV}$. Similarly, the Fe K $\alpha$ lines in HMXBs studied by Torrejón et al. (2010) are also very narrow (unresolved by Chandra). Thus, both theoretical treatments of disk atmospheres and observations of accretion disk corona sources show that narrow, not broad, lines originate there.

In conclusion, we do not find any significant correlation between broad iron line width and the column density of warm absorbers in neutron star low-mass X-ray binaries. Thus, we do not currently find any observational evidence pointing toward line broadening due to a disk wind. Further observations and detailed photoionization modeling of neutron star LMXBs displaying absorption is needed to further test the disk wind scenario for iron line broadening.

We thank Andy Fabian, Chris Reynolds and Rubens Reis for helpful discussions and comments.

\section{REFERENCES}

Barret, D. 2013, ApJ, 770, 9

Beloborodov, A. M. 1999, ApJL, 510, L123

Boirin, L., Méndez, M., Díaz Trigo, M., Parmar, A. N., \& Kaastra, J. S. 2005, A\&A, 436, 195

Brenneman, L. W., \& Reynolds, C. S. 2006, ApJ, 652, 1028

Cackett, E. M., Altamirano, D., Patruno, A., et al. 2009, ApJL, 694, L21
Cackett, E. M., Fabian, A. C., Zogbhi, A., et al. 2013, ApJL, 764, L9

Cackett, E. M., Miller, J. M., Ballantyne, D. R., et al. 2010, ApJ, 720, 205

Cackett, E. M., Miller, J. M., Bhattacharyya, S., et al. 2008, ApJ, 674, 415

D’Aì, A., di Salvo, T., Ballantyne, D., et al. 2010, A\&A, 516, A36

Dauser, T., Wilms, J., Reynolds, C. S., \& Brenneman, L. W. 2010, MNRAS, 409, 1534

De Marco, B., Ponti, G., Cappi, M., et al. 2013, MNRAS, 431, 2441

Díaz Trigo, M., Sidoli, L., Boirin, L., \& Parmar, A. N. 2012, A\&A, 543, A50

Diaz Trigo, M., Sidoli, L., Parmar, A., \& Boirin, L. 2010, in AIP Conf. Proc. 1248, X-Ray Astronomy 2009; Present Status, Multi-Wavelength Approach and Future Perspectives, ed. A. Comastri, L. Angelini, \& M. Cappi (Melville, NY: AIP), 153

Emmanoulopoulos, D., McHardy, I. M., \& Papadakis, I. E. 2011, MNRAS, 416, L94

Fabian, A. C., Zoghbi, A., Ross, R. R., et al. 2009, Natur, 459, 540

Frank, J., King, A. R., \& Lasota, J.-P. 1987, A\&A, 178, 137

Fürst, F., Suchy, S., Kreykenbohm, I., et al. 2011, A\&A, 535, A9

George, I. M., \& Fabian, A. C. 1991, MNRAS, 249, 352

Jimenez-Garate, M. A., Raymond, J. C., Liedahl, D. A., \& Hailey, C. J. 2001, ApJ, 558, 448

Jimenez-Garate, M. A., Raymond, J. C., Liedahl, D. A., \& Hailey, C. J. 2005, ApJ, 625, 931

Kallman, T. R., Angelini, L., Boroson, B., \& Cottam, J. 2003, ApJ, 583, 861

Kara, E., Fabian, A. C., Cackett, E. M., Miniutti, G., \& Uttley, P. 2013a, MNRAS, 430, 1408

Kara, E., Fabian, A. C., Cackett, E. M., et al. 2013b, MNRAS, 428, 2795

Laurent, P., \& Titarchuk, L. 2007, ApJ, 656, 1056

Lin, D., Remillard, R. A., \& Homan, J. 2010, ApJ, 719, 1350

Markoff, S., Falcke, H., \& Fender, R. 2001, A\&A, 372, L25

Miller, J. M. 2007, ARA\&A, 45, 441

Miller, J. M., Maitra, D., Cackett, E. M., Bhattacharyya, S., \& Strohmayer, T. E. 2011, ApJL, 731, L7

Miller, J. M., Raymond, J., Fabian, A., et al. 2006, Natur, 441, 953

Miller, J. M., Raymond, J., Fabian, A. C., et al. 2012, ApJL, 759, L6

Miller, J. M., Raymond, J., Reynolds, C. S., et al. 2008, ApJ, 680, 1359

Miller, J. M., Reynolds, C. S., Fabian, A. C., Miniutti, G., \& Gallo, L. C. 2009a, ApJ, 697, 900

Miller, L., Turner, T. J., \& Reeves, J. N. 2009b, MNRAS, 399, L69

Misra, R., \& Sutaria, F. K. 1999, ApJ, 517, 661

Nandra, K., O'Neill, P. M., George, I. M., \& Reeves, J. N. 2007, MNRAS, 382,194

Papitto, A., Di Salvo, T., D’Aì, A., et al. 2009, A\&A, 493, L39

Ponti, G., Fender, R. P., Begelman, M. C., et al. 2012, MNRAS, 422, L11

Reynolds, C. S., Fabian, A. C., Brenneman, L. W., et al. 2009, MNRAS, 397, L21

Reynolds, C. S., \& Wilms, J. 2000, ApJ, 533, 821

Sim, S. A., Long, K. S., Miller, L., \& Turner, T. J. 2008, MNRAS, 388, 611

Sim, S. A., Miller, L., Long, K. S., Turner, T. J., \& Reeves, J. N. 2010a, MNRAS, 404, 1369

Sim, S. A., Proga, D., Miller, L., Long, K. S., \& Turner, T. J. 2010b, MNRAS, 408, 1396

Sunyaev, R. A., \& Titarchuk, L. G. 1980, A\&A, 86, 121

Titarchuk, L., Laurent, P., \& Shaposhnikov, N. 2009, ApJ, 700, 1831

Torrejón, J. M., Schulz, N. S., Nowak, M. A., \& Kallman, T. R. 2010, ApJ, 715,947

Ueda, Y., Murakami, H., Yamaoka, K., Dotani, T., \& Ebisawa, K. 2004, ApJ, 609,325

Uttley, P., Wilkinson, T., Cassatella, P., et al. 2011, MNRAS, 414, L60

Walton, D. J., Nardini, E., Fabian, A. C., Gallo, L. C., \& Reis, R. C. 2013, MNRAS, 428, 2901

Xiang, J., Lee, J. C., Nowak, M. A., Wilms, J., \& Schulz, N. S. 2009, ApJ, 701, 984

Zoghbi, A., Fabian, A. C., Reynolds, C. S., \& Cackett, E. M. 2012, MNRAS, 422, 129

Zoghbi, A., Reynolds, C., Cackett, E. M., et al. 2013, ApJ, 767, 121 